\title{
Management of Traumatic Inflammatory External Root Resorption
}

\author{
Shikha Bantawa, ${ }^{1}$ Navin Agrawal, ${ }^{1}$ Mannu Vikram, ${ }^{1}$ Vimmi Singh, ${ }^{1}$ Ashok Ayer, ${ }^{1}$ Arbind Rai, ${ }^{1}$ \\ Sushmita Shrestha ${ }^{1}$ \\ ${ }^{1}$ Department of Conservative Dentistry and Endodontics, College of Dental Surgery, BP Koirala Institute of Health \\ Sciences, Dharan, Nepal.
}

\begin{abstract}
Inflammatory external root resorption is one of the major complications after traumatic dental injury. It is characterized by the loss of mineralized dental tissue which may, ultimately, result in loss of the tooth. However, with appropriate treatment, prognosis for these teeth may be greatly improved, by preventing or arresting resorption. This is a case report of an 18-year-old male patient presented with root canal treated maxillary central incisors associated with pain; with history of trauma. Radiographic examination revealed periradicular lesion and associated pathologic resorption of the apical region of the root with respect to 11 and calcification of the root canal with inadequate obturation with respect to 21 . After removal of the root canal filling, 11 was disinfected with intracanal double antibiotic paste. After 2 weeks, antibiotic dressing was removed, and apical third was sealed with mineral trioxide aggregate (MTA). Canal was obturated with custom-fit gutta-percha and accessory cones with AH plus sealer and restoration was done with composite resin. Retreatment was also done with respect to 21 and obturated with custom-fit gutta-percha. Both teeth were restored with E-max crown. No clinical symptoms were apparent, significant osseous healing of the periradicular region was observed with arrest of external root resorption with respect to 11 and no periapical changes were apparent with respect to 21 on subsequent follow-up.
\end{abstract}

Keywords: double antibiotic paste; external root resorption; mineral trioxide aggregate.

\section{INTRODUCTION}

Oral trauma comprises $5 \%$ of all injuries. ${ }^{1}$ As a sequelae of oral trauma: cracks, fracture, avulsion, pulp necrosis, root resorption, root canal obliteration, damage to permanent tooth buds, etc can occur. Inflammatory external root resorption (IERR) is one of the most common complications after traumatic dental injury. Longitudinal studies show that the prevalence of root resorption after trauma is very high $(8-50 \%) .{ }^{2,3}$ Traumatic injuries resulting in reduction or severance of pulpal blood supply, followed by bacterial invasion of the pulp, are also prone to cause damage to the cemental protection of root surfaces. This allows dentinal tubules to become pathways for bacterial toxins to trigger osteoclastic activity externally. ${ }^{4}$

Clinically, teeth are usually asymptomatic. However, as IERR progresses, the teeth may become symptomatic and periradicular abscess may develop which increases tooth mobility. If there is no intervention, the resorptive process may lead to rapid tooth substance loss. However, with appropriate treatment, prognosis for these teeth may be greatly improved, by preventing or arresting resorption. The aim of the present case report is to describe the clinical management of progressive apical root resorption treated 6 years after dental trauma and primary endodontic treatment.

\section{CASE REPORT}

An 18-year-old male presented to the Department of Conservative Dentistry and Endodontics, BPKIHS with a chief complaint of discoloration in upper front teeth (Figure 1a,b). A history of trauma to upper front teeth 6yrs back was present. The patient reported that after one year of trauma he had

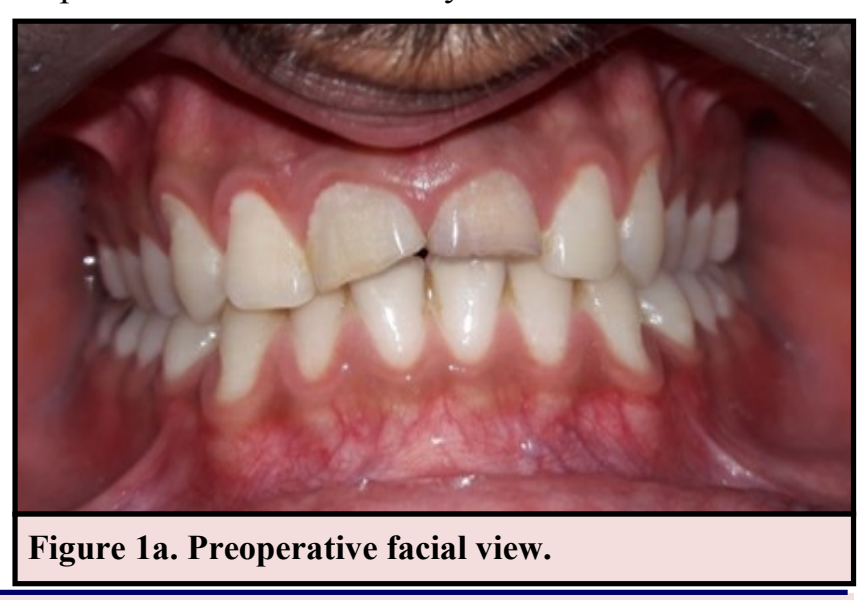

Correspondence: Dr. Shikha Bantawa, Department of Conservative Dentistry and Endodontics, BP Koirala Institute of Health Sciences, Dharan, Nepal. Email: candleshikha@gmail.com. Phone: +977-9842085124. DOI: 10.3126/ jcmsn.v15i1.22238. Article received: 2019-01-05. Article accepted: 2019-02-15. 


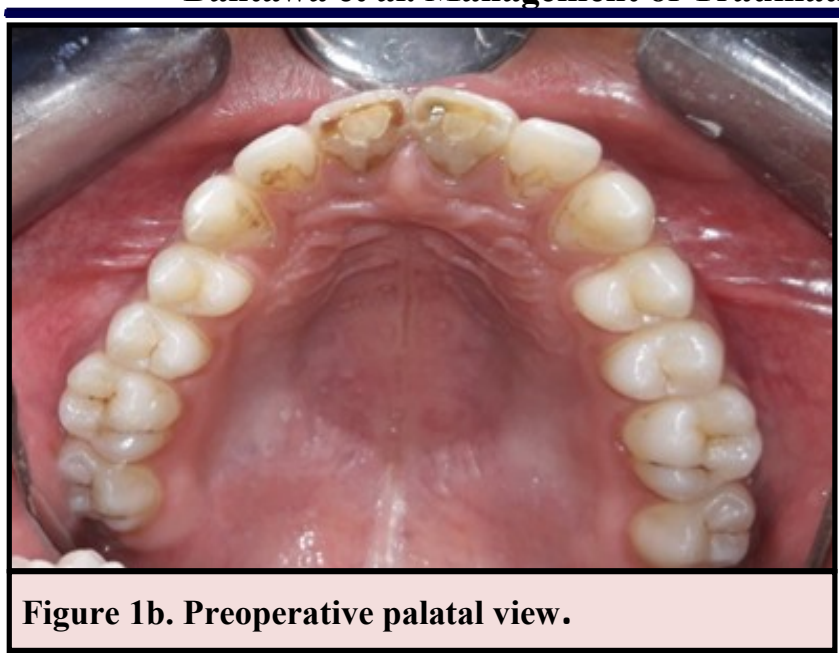

undergone root canal treatment of two front teeth by a dentist. On intraoral examination, 11 and 21 was fractured as well as discolored. Tooth 11 was tender on percussion. Radiographic examination revealed periradicular lesion and associated pathologic resorption of the apical region of the root with respect to 11 and calcification of the root canal with inadequate obturation with respect to 21 (Figure 2).

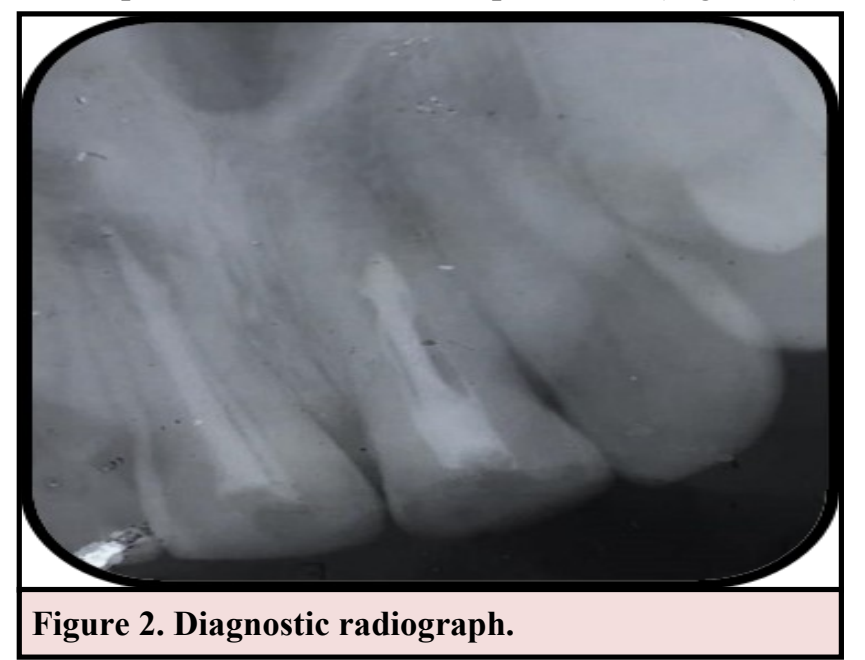

A diagnosis of traumatic inflammatory external root resorption was made with respect to 11 and calcific metamorphosis with inadequate obturation with respect to 21. Revision of treatment was planned. Under rubber dam isolation gutta purcha was removed and working length was determined in relation to 11 and 21 (Figure 3). Debridement of the canal was carried out using irrigants; $2.5 \%$ sodium hypochorite and normal saline. Double Antibiotic Paste dressing was given for disinfection of 11 and patient was recalled after 2 weeks. On subsequent visit, the tooth was no longer tender on percussion. Under rubber dam isolation, collagen material (Kolspon, Eucare Pharmaceuticals Pvt. Ltd., India) was placed as a matrix material before placing mineral trioxide aggregate (MTA) into the canal of 11. Approximately $4 \mathrm{~mm}$ of MTA (Angelus,

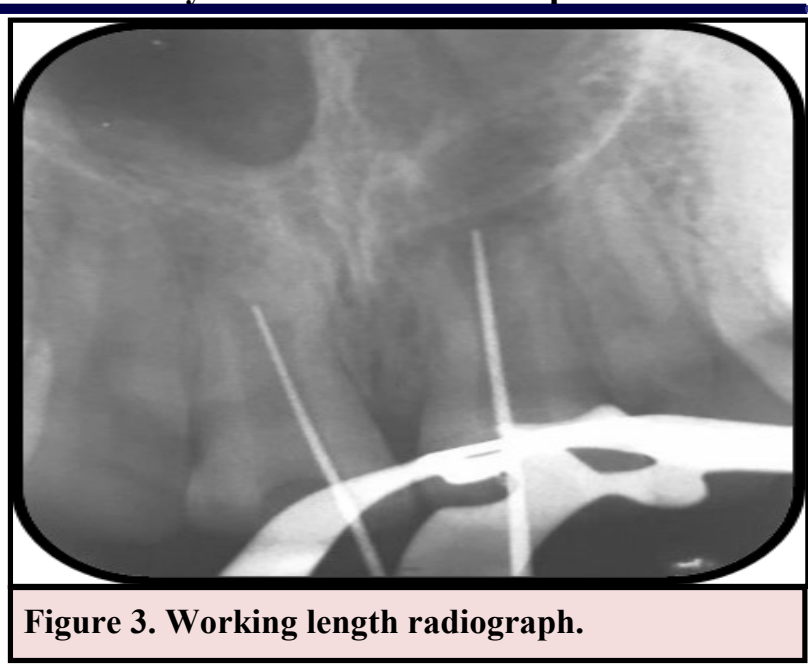

Londrina, Brazil / Clinician's Choice, New Milford, CT) was placed apically (Figure 4) then

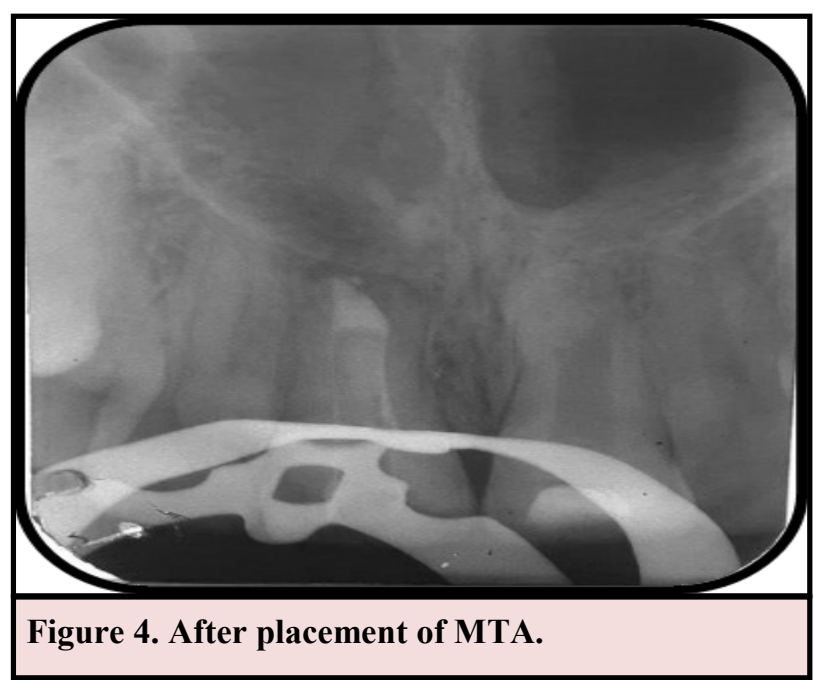

wet paper points were placed in the canal. The canal was sealed with glass ionomer cement and recalled for obturation the following day. Custom fit gutta purcha was prepared and master cone radiograph of 11 and 21 was taken (Figure 5).

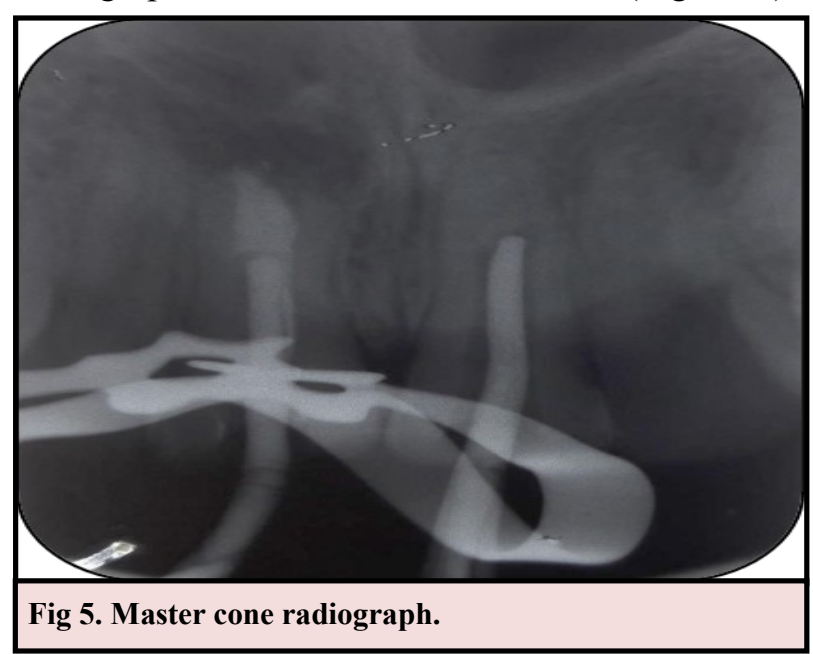

Obturation was done with $\mathrm{AH}$ Plus sealer and access cavity was sealed with direct composite 
restoration. (Figure 6). Tooth preparation was done for all ceramic crown (Figure 7a, b). Cementation
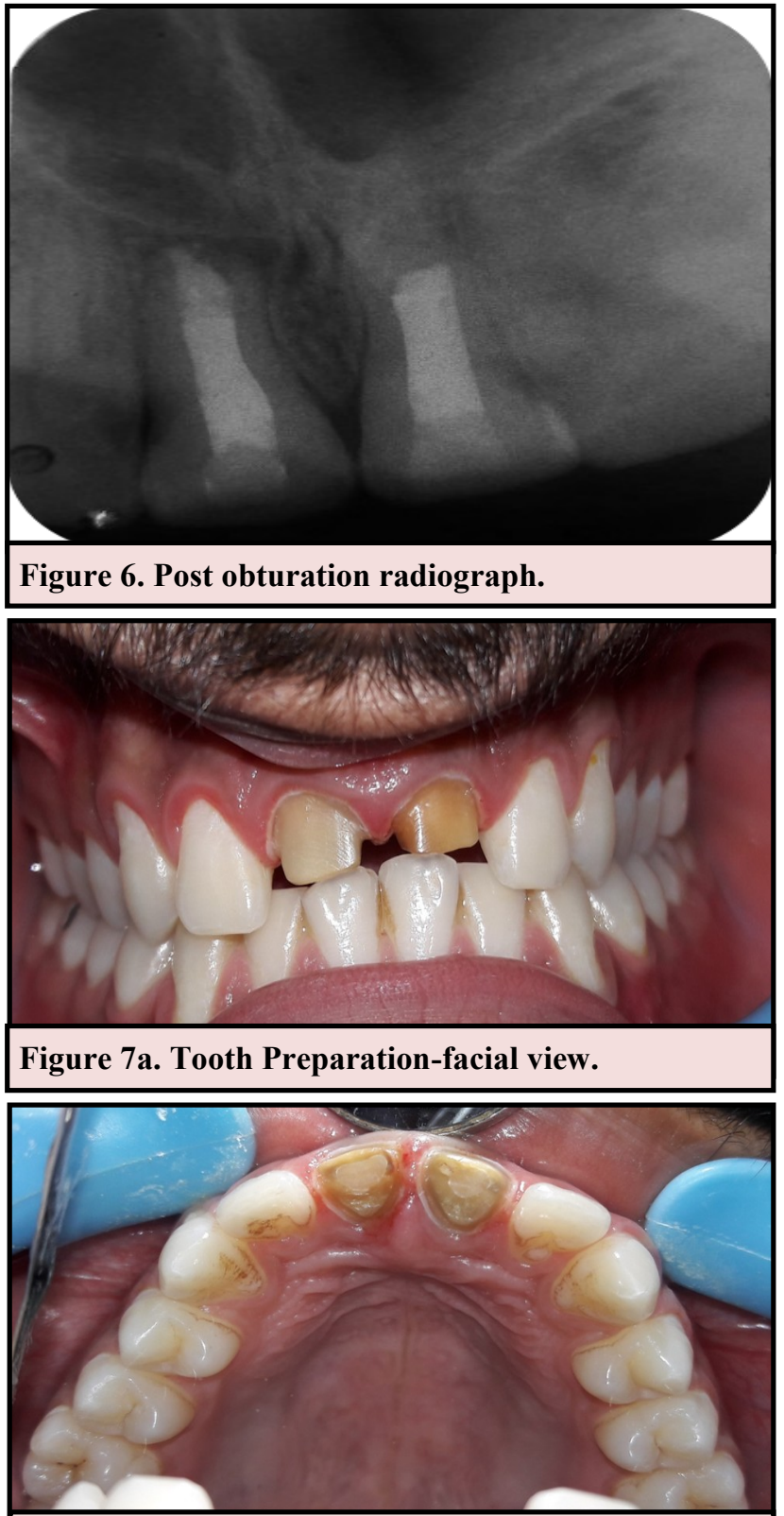

Figure 7b. Tooth Preparation-palatal view.

of E- Max crown was done with resin cement (Figure 8). Six months and one year follow up

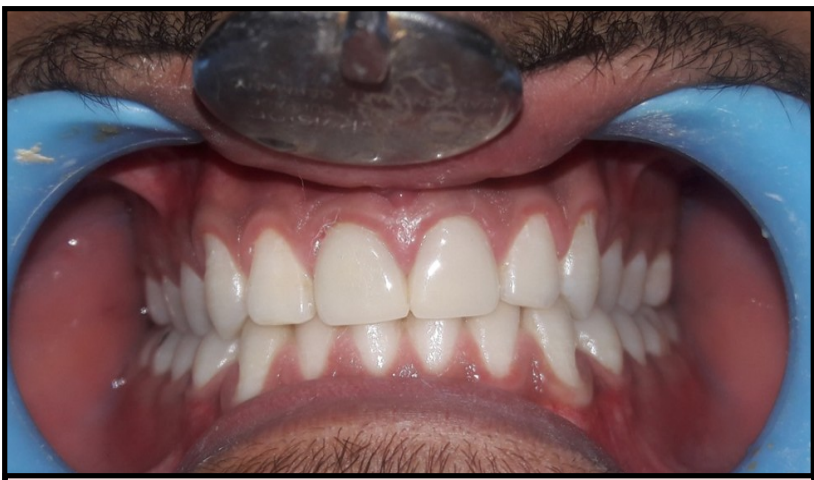

Fig 8a. After cementation of crown: facial view.

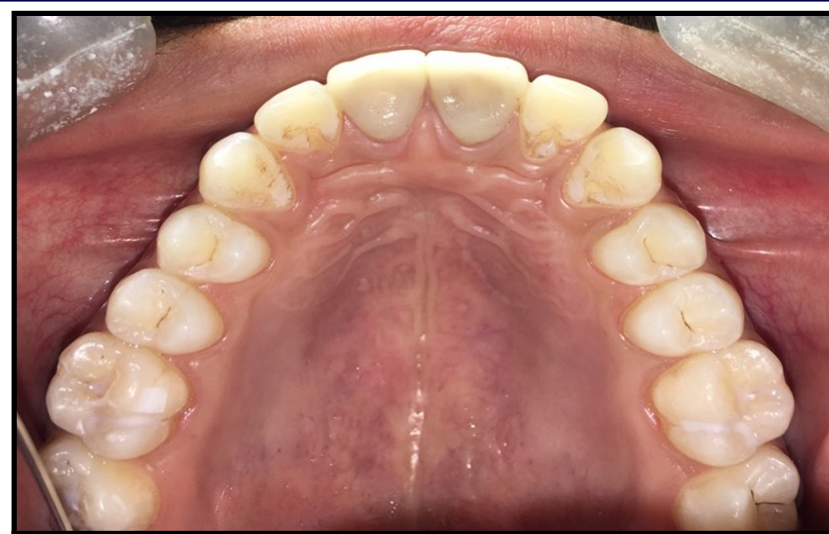

Figure 8b. After cementation of crown: palatal view.

radiograph revealed significant osseous healing and arrest of periapical root resorption in relation to 11 and no periapical changes in relation to 21 (Figure $9,10)$. Patient was asymptomatic and revealed no painful response on percussion.

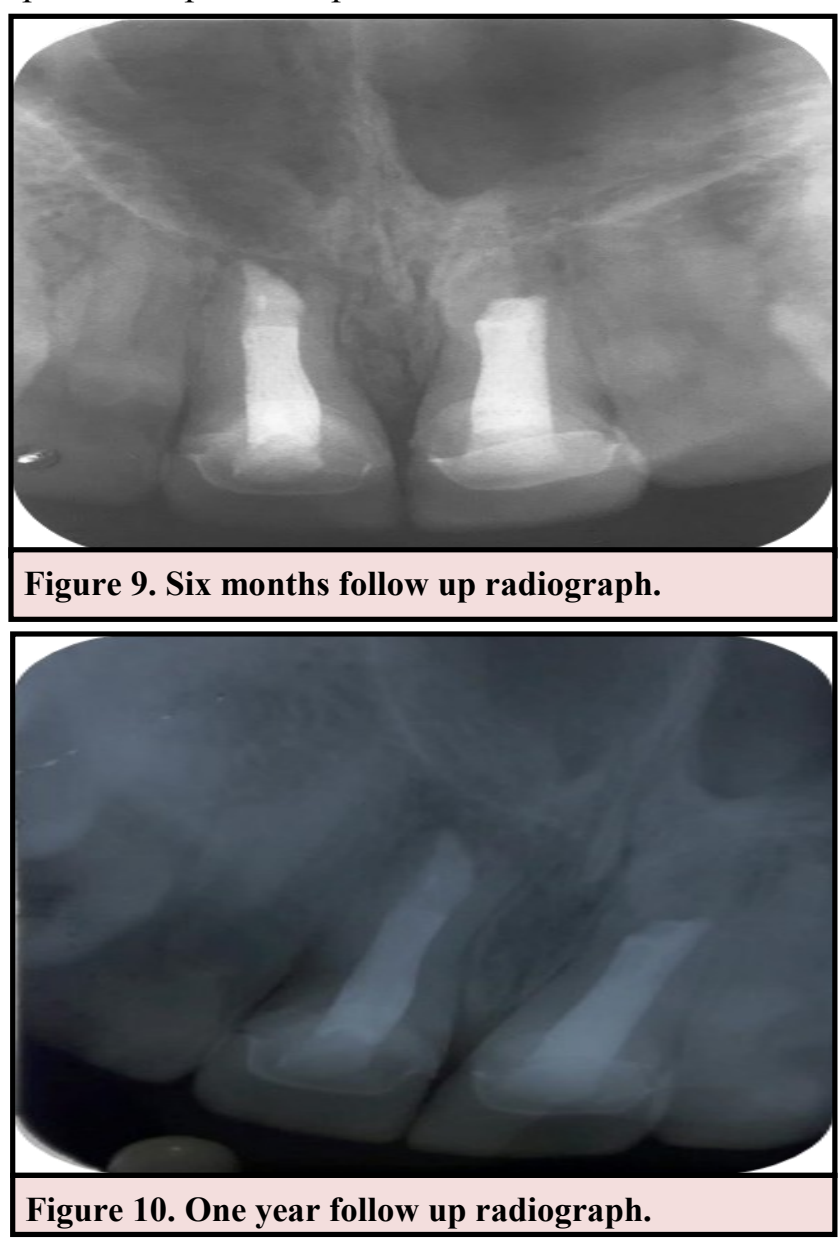

\section{DISCUSSION}

For the management of root resorption, early detection is the key to success because, if detected early, the resorption process can be halted. The challenge of successful endodontics facilitating cessation of resorption is the need for efficient debridement and disinfection followed by optimal 
apical seal. ${ }^{5}$ This case showed previous failure of primary endodontic treatment which was inadequately obturated that lead to poor apical seal. Thus, to obtain complete disinfection and optimal apical seal: double antibiotic paste and mineral trioxide aggregate were used respectively.

Double antibiotic paste (DAP) consist of Metronidazole and Ciprofloxacin. Metronidazole is selectively toxic to anaerobic microorganisms. It binds to the DNA, disrupts the helical structure and lead to rapid cell death. Ciprofloxacin is a synthetic floroquinolone which inhibits bacterial DNA gyrase enzyme and shows rapid bactericidal action. It exhibits very potent activity against gram negative bacteria but very limited activity against gram positive bacteria. Most of the anaerobic bacteria are resistant to ciprofloxacin. Hence in treating polymicrobial infections, it is often combined with metronidazole. ${ }^{6}$ It is the modification of triple antibiotic paste where Minocycline has been removed due to its side effect of staining tooth. ${ }^{7}$ When compared with calcium hydroxide, DAP are more effective in eliminating Enterococcus faecalis which are more dominant in persistent infection even after endodontic treatment. ${ }^{8}$ In the case of apical root resorption, there is a need to limit the material to the apex so small pieces of collagen membranes were packed against which MTA can be condensed. MTA has several desirable properties in terms of its biocompatibility, bioactivity, hydrophilic, radioopacity, sealing ability and low solubility. ${ }^{9}$ MTA has the ability to form hydroxyapatite like layer when it comes in contact with physiologic fluid having good sealing property and encourages regeneration of periodontal ligament, bone and cementum making it choice of material in the management of root resorption. 5,10

Six months and one year follow up of this case revealed favorable clinical and radiographic outcome, with the evidence of arrest of the resorptive process and increased bone formation. This result supported the suitability of the use of this method in the management of root resorption. Similar case reports, using MTA in the successful treatment of root resorption has been documented. ${ }^{11-13}$

\section{CONCLUSIONS}

Key to the success of management of inflammatory external root resorption is the early diagnosis followed by complete debridement, disinfection and good apical seal.

\section{REFERENCES}

1 Andersson L. Epidemiology of traumatic dental injuries. JOE. 2013; 39: S2-S5. https:// doi.org/10.1016/j.joen.2012.11.021

2. Santos BO, Mendonca DS, Sousa DL, Moreira Neto JJ, Araújo RB. Root resorption after dental traumas: classification and clinical, radiographic and histologic aspects. RSBO (Online). 2011 Dec;8 (4):439-45.

3. Finucane D, Kinirons MJ. External inflammatory and replacement resorption of luxated, and avulsed replanted permanent incisors: a review and case presentation. Dental Traumatology. 2003; 19: 170-4. PMID:12752540

4. Bakland LK, Andreasen JO. Dental traumatology: essential diagnosis and treatment planning. Endodontic topics. 2004; 7: 14-34. 10.1111/j.16011546.2004.00059.x PMID: 20433980

5. Ashwini T, Hosmani N, Patil CR and Yalgi VS. Role of mineral trioxide aggregate in management of external root resorption. Journal of conservative dentistry: JCD. 2013; 16: 579-81. 10.4103/09720707.120937

6. Parasuraman VR and Muljibhai B. 3MixMP in endodontics an overview. J Dent Med Sci. 2012; 3: 36-45.

7. Trope M. Treatment of the immature tooth with a non-vital pulp and apical periodontitis. Dental

Clinics. 2010; 54: 313-24. https://doi.org/10.1016/ j.cden.2009.12.006.

8. Ravi K. Antimicrobial Efficacy of Various Intracanal Medicaments against Enterococcus faecalis. Journal of Pharmaceutical Sciences and Research. 2017; 9: 1861-3.

9. Tawil PZ, Duggan DJ and Galicia JC. MTA: a clinical review. Compendium of continuing education in dentistry (Jamesburg, NJ: 1995). 2015; 36: 247-64. PMID: 25821936

9. Torabinejad M, Hong C-U, Lee S-J, Monsef M and Ford TRP. Investigation of mineral trioxide aggregate for root-end filling in dogs. Journal of Endodontics. 1995; 21: 603-8. 10.1016/S0099-2399(06)81112-X PMID: 8596081

11.Kuo T-C, Cheng Y-A and Lin C-P. Clinical management of severe external root resorption. CHINESE DENTAL JOURNAL. 2005; 24(1): 59-63.

12.Kusgoz A, Yildirim $\mathrm{T}$, Alp $\mathrm{CK}$ and Tanriver $\mathrm{M}$. Management of root resorption with mineral trioxide aggregate complicated by a luxation injury: report of a case with six-year follow-up. The Journal of the Pakistan Medical Association. 2017; 67: 134-6.

13.Vineet SA and Sonali K. Clinical management of severe external root resorption and immature open apex with MTA and calcium hydroxide-A case report. Endodontology [online]. 2013: 83-8.

Citation: Bantawa S, Agrawal N, Vikram M, Singh V, Ayer A, Rai A, Shrestha S. Management of Traumatic Inflammatory External Root Resorption. JCMS Nepal. 2019;15(1):71-4. 